Preprint SPBU-IP-98-9

\title{
Effective Theories with Maximal Analyticity
}

\author{
Alexander V. Vereshagin, Vladimir V. Vereshagin 七 $^{\prime}$
}

\author{
Theoretical Physics Department \\ St.Petersburg State University \\ Ulyanovskaya st 1 \\ 198904 St.Petersburg \\ Russia
}

\begin{abstract}
In this paper (second in the series) we study the properties of tree-level binary amplitudes of the infinite-component effective field theory of strong interaction obeying the requirements of quark-hadron duality and maximal analyticity. In contrast to the previous paper, here we derive the results following from less restrictive - Regge-like - boundedness conditions. We develop the technique of Cauchy's forms in two variables and show the string-like structure of a theory. Next, we derive the full set of bootstrap constraints for the resonance parameters in $(\pi, K)$ system. Numerical test shows: (1) those constraints are consistent with data on well established vector resonances; (2) two light broad resonances $-\sigma$ and $\kappa$ - are needed to saturate sum rules following from Chiral symmetry and analyticity. As a by-product we obtain expressions for the parameters of Chiral expansions and give corresponding estimates.
\end{abstract}

\footnotetext{
${ }^{1} e$-mail: vvv@av2467.spb.edu
} 


\section{Introduction}

In a previous paper by one of us [1] it was shown that the requirements of meromorphy and polynomial boundedness applied to the most general form of tree-level amplitude of a given binary process give rise to a certain infinite system of constraints for coupling constants and particle masses. Also it was pointed out that those constrains realize the so-called bootstrap conditions which - in turn - mirror the dual properties of hadronic amplitudes.

Some of the bootstrap conditions for $(\pi, K)$ scattering amplitude derived in ref [1] can be checked numerically because the modern experimental data provide the necessary values for masses and coupling constants. Since the conditions in question take a form of rapidly converging sum rules (SR), one can select for numerical check those SR which can be saturated (with sufficient accuracy) by the contributions of few lightest resonances.

After the checking of several SR we have recognized the presence of systematical discrepancies between their left and right hand sides; those discrepancies could be hardly explained by incompleteness of modern database. The point is that - roughly speaking our analysis has shown that the relative magnitude of the two most significant contributions (those of $\rho$ - and $K^{*}-$ mesons) following from SR derived in [1] were inconsistent with the well established values of the corresponding masses and coupling constants.

This observation shows that the system of postulates accepted in [1] is inconsistent with the physical reality and, hence, it must be reconsidered. This is done in a given paper. It is shown that replacement of the decreasing asymptotics requirement for the inelastic channel amplitude by the Regge conditions results in a new system of bootstrap constrains which is quite reasonable from the phenomenological point of view.

The paper is organized as follows. In Sec. 2 we explain the essence of the actual physical problem which general solution this paper is devoted to. Besides, we explain also the constructive formulation of the maximal analyticity principle which plays a key role in our approach. Sec. 3 is the central one: here we give the general outline of the mathematical tool specially constructed to work with meromorphic functions of two (and more) variables with fiberwise given asymptotics. Since in [1] it is shown that our approach gives rise to certain duality properties, in Sec. 4 we consider the widely known example of the dual (string) amplitude constructed from a single $B$-function. This analysis allows us to point out some particular suggestions implicitly contained in conventional dual hadron amplitudes. In Sec. 5 we apply the developed technique to derive the set of bootstrap constraints for the parameters of $(\pi, K)$ amplitude, this set basing on weaker suggestions (compared to those accepted in [1]) about the asymptotics of inelastic channel. In contrast with [1] we show here the explicit form of generating functions allowing us to write down (in Sec. 6) several rapidly converging bootstrap 
conditions (sum rules) which can be easily checked with the help of known data on spectrum parameters. After the checking of those SR validity, in Sec. 7 we derive explicit expressions for low energy parameters and compute the corresponding numerical values. Besides, we show that our SR require the existence of light scalar resonances with isospins $I=0,1 / 2$ and estimate their parameters. At last, Sec. 8 is devoted to the discussion of the results obtained. Appendix contains the necessary formulae and relations.

\section{Preliminary notes}

First of all we would like to recall the essence of the problem which stimulated us to begin a systematic study of the properties of tree-level amplitudes in a framework of effective field theory approach. This is the widely discussed problem of low energy coefficients (LEC's) appearing in chiral expansions [2, 3, 4] (the excellent discussion can be found in [5]). Those coefficients cannot be fixed with a help of the symmetry constraints since they are nothing but coupling constants corresponding to various invariant interaction lagrangians. The number of LEC's very rapidly increases with the expansion order. This very circumstance creates a problem because it reduces to zero the predictive power of Chiral perturbation theory (ChPT) in higher orders. That is why it would be very interesting to find a way allowing one to fix the LEC's or, at least, to restrict their values.

Clearly, to solve this problem we have to take account of certain new principles. It would be the best if we take advantage of the principles which are no less general than those used as the basis for the effective theory.

Following [1], we study here the possibility to attract for this purpose the suitably formulated old good principle of maximal analyticity along with the polynomial boundedness requirement for tree-level amplitude (first suggested in [6]; see also [7]). Some arguments (as well as the corresponding list of references) in favor of the latter requirement are given in [目]. Thus we need to explain here in more detail both the motivation and the exact formulation of the maximal analyticity principle. The best way to do this is to consider the simple example: the low energy elastic scattering of two identical pseudoscalar particles with the mass $\mu \ll m_{1}$, where $m_{1}$ stands for the mass of the lightest allowed resonance. In this case the low energy effective tree-level amplitude takes a form

$$
A(s, t, u)=\sum_{i, j, k} a_{i j k} s^{i} t^{j} u^{k}
$$

Here summation in $i, j, k$ is infinite by the very meaning of the term "effective", $a_{i j k}$ is completely symmetric in its indices and the problem of LEC's is precisely that of 
$a_{i j k}$. To the first glance these constants seem to be free parameters of our effective theory. However, this - widely believed - point of view is not quite correct. Below we demonstrate that certain limitations on the values of $a_{i j k}$ follow directly from the natural requirements of analyticity.

Here it is pertinent to recall one of the basic principles of $S$-matrix theory, namely, the maximal analyticity principle. It says, that the only singularities of a given process amplitude are those required by the unitarity relation. In the framework of field theory approach this relation is realized perturbatively via the loop expansion scheme. This scheme automatically generates all necessary singularities required by unitarity. Besides, it might develop also the unnecessary singular structures if the corresponding terms are contained in the tree-level amplitudes.

Thus we conclude that, to avoid a contradiction with the maximal analyticity principle, one has to take the tree-level amplitudes as regular as possible.

Clearly, the singular structure of tree-level amplitudes is a matter of model. The effective amplitude of the elastic photon-photon scattering provides an example when this structure is very complicated. However, it is well known that this feature is uniquely connected with the existence of electron which - together with photon - has to be taken into account when constructing the full system of states in QED. If the electron field is included in Lagrangian as a separate degree of freedom, the analytical structure of the lowest order amplitudes becomes simple, the photon-photon scattering appearing as one of the higher-order processes.

Extremely interesting analysis of the similar effect in a framework of "toy-theory" the quark-level linear $\sigma$-model — has been implemented in the recent paper [8]. The authors show that the double counting problem (appearing due to dynamically generated additional scale) can be solved using the compositeness condition (see Chapter 10 in [5]), the result providing a natural self-consistent field-theoretic interpretation in terms of either elementary particle or the bound state.

Now, let us come back to the tree-level amplitude (1). To fix its singular structure we can rely on the hypothesis of quark-hadron duality which says that the full set of colorless quark-gluon states is equal to the full set of hadronic states. This can be also formulated as follows: the functional integral for $S$-matrix in QCD can be identically rewritten in terms of hadronic fields. Leaning upon this statement one concludes that the singular structure of tree-level amplitude (1) is completely determined by the contributions of relevant one-particle hadronic states. In other words, the quark-hadron duality together with maximal analyticity principle require of the tree-level amplitude of a given binary process to be a meromorphic function of 3 (dependent) Mandelstam variables, the only allowed singularities being just simple poles and the ambiguity points (see Sec. 3 below).

The above reasoning allows us to avoid the refereeing to the large- $N_{c}$ limit of QCD 
(cf. with [9]). Moreover, it allows one to reduce the very difficult (from the purely phenomenological point of view) problem of LEC's to the problem of spectrum parameters - on-shell triple couplings and masses (see [1]).

\section{Cauchy's form in the case of two variables}

The main tool used in [1] to carry out the analytic continuation, connecting the direct- and cross-chanel tree-level amplitudes, is based on the Mittag-Leffler theorem in its constructive form provided by the Cauchy method. This method allows one to write down a general expression (which we call below as Cauchy's form) for the polynomially bounded meromorphic function $f(z)$ of one complex variable $z$, with given poles $p_{n}(n=1, \ldots)$, corresponding principle parts $g_{n}(z)$ and the degree $N$ of bounding polynomial. This expression reads

$$
f(z)=\sum_{n=0}^{N} \frac{1}{n !} f^{(n)}(0) z^{n}+\sum_{p=1}^{\infty}\left[g_{p}(z)-h_{p}^{(N)}(z)\right] .
$$

Here

$$
h_{p}^{(N)}(z) \equiv \sum_{n=0}^{N} \frac{g_{p}^{(n)}(0)}{n !} z^{n},
$$

are the so-called correcting polynomials needed to ensure the convergence of the infinite sum of pole contributions. It is implied that $f(0)$ is regular, otherwise, the corresponding principal part $g_{0}(z)$ should be added to the right hand side of the Eq. (21).

A rigorous proof of the form (2) can be found in textbooks on complex analysis (see, e.g., [10, 11]). However, in our work we use the generalized version of (2) allowing one to consider meromorphic functions of two complex variables $(\nu, x)$. As far as we know, such a form could hardly be found in the literature. Therefore, it makes sense to give here a sketch of the proof of the generalized version of (2) most suitable for our needs. Later on we imply that the reader is familiar with the case of one complex variable.

First of all we would like to remind the reader, that every meromorphic function of two (and more) complex variables $f\left(z_{1}, z_{2}\right)$ has two different kinds of singularities: poles and the ambiguity points. The last term can be best explained by the following example. Consider

$$
f\left(z_{1}, z_{2}\right)=\frac{z_{1}}{z_{2}} .
$$

This is a meromorphic function, its polar set being the hyperplane $\left(z_{1}, 0\right)$ except the point $(0,0)$ which is precisely the ambiguity point. The value of $f\left(z_{1}, z_{2}\right)$ at this point depends 
of the path chosen to reach it. For example,

$$
\lim _{z_{2} \rightarrow 0} \lim _{z_{1} \rightarrow 0} f\left(z_{1}, z_{2}\right)=0
$$

while

$$
\lim _{z_{1} \rightarrow 0} \lim _{z_{2} \rightarrow 0} f\left(z_{1}, z_{2}\right)=\infty .
$$

Less trivial example is provided by Fig.1 where we show the geography of the ambiguity points corresponding to the string-like amplitude (10) considered in Sec. 4 below.

In order to avoid unnecessary complications which have nothing to do with the fieldtheoretical problems considered in our paper, below (except the Sec. 4) we concentrate solely on a consideration of the narrow class of meromorphic functions $f(\nu, x)$ satisfying the following conditions:

- They have only simple poles in each variable.

- They have no poles in both variables simultaneously.

- They have no fixed (i.e. independent of $x$ ) poles in $\nu$; only moving poles of the form

$$
\nu-x=Q_{i} ; \quad \nu+x=-Q_{i}
$$

with

$$
0<Q_{i}<Q_{i+1}, \quad(i=1,2, \ldots)
$$

are allowed.

- In the variable $x$ they have both moving poles of the form (3) and fixed poles at the points

$$
x=M_{i}^{2}, \quad(i=1,2, \ldots)
$$

where

$$
0<M_{i}^{2}<M_{i+1}^{2} .
$$

Nevertheless, it should be noted that the analysis of more involved cases creates no difficulties.

To construct the generalized Cauchy form in $\nu$ (at fixed $x$ ) we need to know asymptotics of $f(\nu, x)$ at large $\nu$. Since it might depend on $x$, we have to consider the ratio

$$
\frac{f(\nu, x)}{\nu^{D(x)}}
$$


at large $\nu$. In the most interesting for us case, when the Regge asymptotic condition is imposed (see [四])

$$
D(x)=\alpha+\beta x
$$

Let us introduce the step function

$$
N_{x} \equiv E[D(x)]+1
$$

where $E[y]$ stands for the maximal integer less or equal to $y$. This allows one to draw on the conventional definition of polynomial boundedness (see, e.g., [10, 11]). Indeed, let us consider real $x$ from a small interval $[a, b]$ such that

$$
N_{a}=N_{x}=N_{b} \equiv N
$$

We say that the meromorphic function $f(\nu, x)$ is polynomially bounded in $\nu$ in the band

$$
B_{x}\{|\nu|<\infty, x \in[a, b]\}
$$

if there is a finite integer $N$ and infinite system of smooth contours $C_{p}$ (circles with the radii $\left.R_{p+1}>R_{p}, p=1,2, \ldots\right)$ in the complex- $\nu$ plane such that

$$
\max _{x \in[a, b] ; \nu \in C_{p}}\left|\frac{f(\nu, x)}{\nu^{N+1}}\right| \equiv M_{p} \stackrel{p \rightarrow \infty}{\longrightarrow} 0 .
$$

The minimal $N$ providing the correctness of the uniform (in $x$ ) estimate (乘) we call as the degree of bounding polynomial in $B_{x}$. This definition is equally applied for both increasing and decreasing asymptotics, $N$ taking the negative values in the latter case. The value $N \leq-1$ corresponds to the superconvergent asymptotic behavior.

It is important to stress that we consider the radii $R_{p}$ to be independent of $x$. With the above definition in hand we can immediately write down the generalized Cauchy form for the meromorphic function $f(\nu, x)$, polynomially bounded (with the degree $N$ ) in the band $B_{x}$. It looks as follows

$$
f(\nu, x)=\sum_{k=0}^{N} \frac{1}{k !} \frac{\partial^{(k)} f(0, x)}{\partial \nu^{k}} \nu^{k}+\sum_{m=1}\left[\frac{r_{m}(x)}{\nu-p_{m}(x)}-h_{m}^{(N)}(\nu, x)\right],
$$

where

$$
h_{m}^{(N)}(\nu, x)=-\frac{r_{m}(x)}{p_{m}(x)} \sum_{k=0}^{N} \frac{\nu^{k}}{p_{m}^{k}(x)} .
$$

The proof is based on a consideration of the following contour integral in the complex- $\nu$ plane:

$$
I_{p}(\nu, x, N)=\frac{1}{2 \pi i} \int_{C_{p}} \frac{\nu^{N+1}}{z^{N+1}} \frac{f(z, x)}{(z-\nu)} d z
$$


Exactly as in the case of one variable, it can be shown that at every fixed $x \in[a, b]$

$$
f(\nu, x)=\sum_{k=0}^{N} \frac{1}{k !} \frac{\partial^{(k)} f(0, x)}{\partial \nu^{k}} \nu^{k}+\sum_{m=1}^{p}\left[\frac{r_{m}(x)}{\nu-p_{m}(x)}-h_{m}^{(N)}(\nu, x)\right]+I_{p}(\nu, x, N) .
$$

Taking the limit $p \rightarrow \infty$ and using the condition (4) one derives from (6) the desired form (5) expressing the function $f(\nu, x)$ of one complex $(\nu)$ and one real $(x)$ variable in the band $B_{x}$ as an expansion in its poles in $\nu$.

¿From the given above sketch one can derive the following conclusions:

1. Each item of the infinite sum in (5) combines the contributions from all the poles confined between $C_{m-1}$ and $C_{m}$, i.e. from those with

$$
R_{m-1}<\left|p_{m}(x)\right|<R_{m}
$$

Otherwise, the convergence of the summation procedure is not guaranteed.

2. At any $x \in[a, b]$ the partial fraction expansion (5) converges uniformly everywhere in the complex- $\nu$ plane except the small open vicinities of poles.

3. At any fixed $\nu$ the form (5) in the band $B_{x}$ can be equally treated as the uniformly (in $x$ ) convergent series of (analytic) functions of $x$. This property makes the expansion (5) a useful tool to carry out the analytic continuation in $x$.

4. The form (5) certainly remains valid if the minimal degree $N$ is changed for any integer $\tilde{N}>N$. In this case, however, each of the series

$$
S_{n} \equiv \sum_{m} \frac{r_{m}(x)}{p_{m}^{n}(x)}
$$

with $n=N+1, \ldots, \tilde{N}$ converges and, hence, it can be summed independently. It is easy to show that

$$
S_{n}+\left.\frac{1}{n !} \frac{\partial^{n} f(\nu, x)}{\partial \nu^{n}}\right|_{\nu=0}=0, \quad(n=N+1, \ldots, \tilde{N})
$$

This, in turn, means that each unnecessarily high degree of $\nu$ taken into account in the correcting polynomials $h_{m}^{(\tilde{N})}(\nu, x)$ is effectively cancelled by the corresponding item appearing simultaneously in the first - regular in $\nu$ - term of (5). Thus we conclude that the Cauchy form (5) presents a well defined rigid construction allowing no twofold interpretation. 
5. With the properly chosen functions $r_{i}(x)$ and $p_{i}(x)$ one can secure the convergence of the series (7) even for $n<N$. However, this does not mean that the equality (8) is also valid for $n<N+1$. The minimality of the declared degree $N$ of a bounding polynomial corresponds to the necessary presence of $\nu^{N}$ either in the regular term in (5) or in correcting polynomials or in both terms simultaneously. In other words, the actual presence of $\nu^{N}$ in (5) mirrors the asymptotic behavior of the type

$$
f(\nu, x) \sim|\nu|^{D(x)}
$$

with $N \leq D(x)<N+1$.

There is an important exception to the above formulated rule, this exception being connected solely with our special choice of the system of contours $C_{p}$ which we took symmetric with respect to the origin of the complex- $\nu$ plane. This choice results in a particular method of summation in (5): each item of the sum over poles contains the contributions of all the poles with the same value of $\left|p_{i}\right|$. In the case when $f(\nu, x)$ is odd (even) in $\nu$, the correcting polynomials are also odd (even). The same is true with respect to the regular term. Thus, in this particular case the correcting polynomials of the degree $N$ ensure the convergence of the partial fraction expansion (5) for the asymptotic low (9) with $D<N+2$, the presence of $\nu^{N}$ corresponding to the asymptotic behavior (9) with $N \leq D<N+2$.

6. If the uniform in $x$ estimate (4) is valid for $x \in[a, b]$ with $N_{a} \neq N_{b}$ one can use the Cauchy form (5) with $N=\max \left\{N_{a}, N_{b}\right\}$ to present the function $f(\nu, x)$ in the band $B_{x}$.

The usefulness of the technique developed in this Section is explained by the fact that meromorphic functions with fiberwise given Regge asymptotics appear naturally in the framework of effective hadron field theory. This very technique (first suggested in [1]) is used throughout the remaining part of our paper.

\section{String amplitudes and background interactions}

The results of the previous section allow us to argue that:

A Every polynomially bounded meromorphic function $f(\nu, x)$ can be presented in a form of the convergent series over its poles in $\nu$ at those values of $x$ which correspond to the decreasing asymptotic behavior in $\nu$ (we mean the contour asymptotics). 


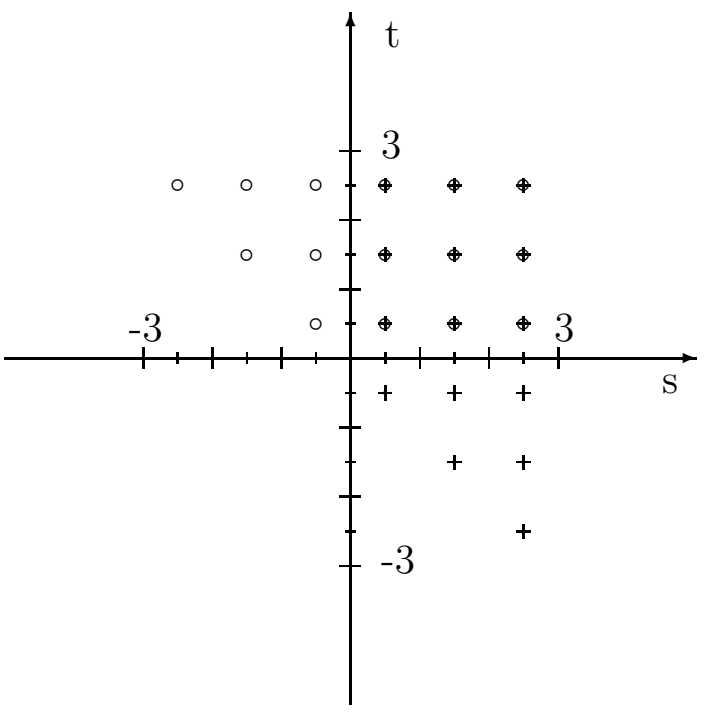

Figure 1: String-like amplitude (10): locations of ambiguity points. o — first series, + - second series, $\oplus$ - superposition of two series (so-called "Odorico zeros").

B On the contrary, at those $x$ which correspond to a constant or increasing asymptotics in $\nu$, none of such functions admit a representation constructed solely of the pole contributions: the appearance of "background" terms (polynomials in $\nu$ with coefficients depending on $x$ ) is inevitable in this case.

In the light of these statements it is extremely instructive to analyze the structure of the famous Veneziano ansatz [12] based on $B$-functions which are widely believed to be constructed solely from resonances (for the review see [13]). By way of illustration we consider the simplest dual (or, the same, string) amplitude without tachyon:

$$
A(s, t)=\left[1-\alpha_{1}(s)-\alpha_{1}(t)\right] B\left\{1-\alpha_{1}(s) ; 1-\alpha_{1}(t)\right\} .
$$

It is implied that kinematical variables are chosen such that

$$
\alpha_{1}(x)=\frac{1}{2}+x
$$

Fig. 1 shows the disposition of ambiguity points of $A(s, t)$; note, that it reflects the space fibering structure corresponding to the asymptotic behavior.

Since the pomeron contribution is not an issue here, one can consider $A(s, t)$ as the amplitude of the process $\pi^{-} \pi^{+} \longrightarrow \pi^{-} \pi^{+}$. Let us study the structure of $A(s, t)$ at $t=0$. 
In this case the actual (radial) asymptotic behavior for $\arg s \neq 0$ follows the Regge low $A(s, 0) \sim s^{1 / 2}$ which is also true with respect to behavior on the system of circles $C_{n}$ with $R_{n}=n$. Hence, according to the results of Sec. 3, the meaningful partial fraction expansion for $A(s, 0)$ cannot be written without introducing of background terms of zeroth order in $\nu$. Let us check this point. For this we need to know the principal parts $g_{n}(s, 0)$ at the poles

$$
p_{n}=n+\frac{1}{2}, \quad n=0,1, \ldots
$$

Rewriting (10) in the form

$$
A(s, 0)=\sqrt{\pi} \frac{\Gamma\left(\frac{1}{2}-s\right)}{\Gamma(-s)}
$$

and using the well-known formulae for $\Gamma$-function, we obtain

$$
g_{n}(s, 0)=\frac{r_{n}(0)}{s-\left(n+\frac{1}{2}\right)},
$$

where

$$
r_{n}(0)=\frac{(2 n+1) ! !}{n ! 2^{n+1}} .
$$

¿From (12) and (13) it follows immediately that the formal sum of principal parts ("nothing else but poles!")

$$
\sum_{n=0}^{\infty} g_{n}(s, 0)
$$

diverges at every point of the complex-s plane. Thus we conclude that, in complete accordance with the statement $B$ above, the dual amplitude (10) at $t=0$ along with the singular part (the sum of pole contributions) contains also certain regular terms. It is not difficult to write down the corresponding convergent expansion. Bearing in mind that $A(0,0)=0$, we have (see Sec. 3):

$$
A(s, 0)=\sum_{n=0}^{\infty}\left[\frac{r_{n}(0)}{s-\left(n+\frac{1}{2}\right)}-\frac{r_{n}(0)}{\left(n+\frac{1}{2}\right)}\right] .
$$

It can be easily shown that the series (14) converges uniformly and absolutely everywhere in the complex-s plane except small open vicinities of poles. This follows from the absolute convergence of the series

$$
\sum_{n=0}^{\infty} \frac{r_{n}(o)}{\left(n+\frac{1}{2}\right)^{2}} \equiv \sum_{n=0}^{\infty} a_{n}
$$


the latter, in turn, can be shown with the help of Gauss's test

$$
\left|\frac{a_{n+1}}{a_{n}}\right|=1+\frac{A}{n}+O\left(\frac{1}{n^{2}}\right),
$$

because in our case $A=-3 / 2<-1$.

The similar analysis for $\mathrm{A}(\mathrm{s},-1)$ shows that the series of principal parts

$$
A(s,-1)=\sum_{n=o}^{\infty} \frac{r_{n}(-1)}{s-\left(n+\frac{1}{2}\right)},
$$

where

$$
r_{n}(-1)=-\frac{(2 n-1) ! !}{n ! 2^{n+1}},
$$

converges by itself (also uniformly and absolutely) and, hence, there is no necessity to take account of any background terms associated with the correcting polynomials. This is precisely the result which one would expect in accordance with the known asymptotic behavior $A(s,-1) \sim s^{-\frac{1}{2}}$. It provides an illustration to the statement $A$.

To fill a gap between $t=0$ and $t=-1$ in the above reasoning we need to account for the explicit dependence of $r_{n}(t)$ and $A(0, t)$ of the variable $t$. In the case under consideration this can be easily done because we can take advantage of the relation

$$
\frac{\Gamma(z) \Gamma(a+1)}{\Gamma(z+a)}=\sum_{n=0}^{\infty} \frac{(-1)^{n}}{n !} a(a-1)(a-2) \ldots(a-n) \frac{1}{z+n},
$$

which is valid for $a>0$. Transforming the left hand side

$$
\frac{\Gamma(z) \Gamma(a+1)}{\Gamma(z+a)}=\frac{(z+a)}{(a+1)} \frac{\Gamma(z) \Gamma(a+2)}{\Gamma(z+a+1)}
$$

and taking

$$
a=-\frac{1}{2}-t, \quad z=\frac{1}{2}-s,
$$

one obtains

$$
A(s, t)=\sum_{n=0}^{\infty}\left[\frac{r_{n}(t)}{s-p_{n}}+\frac{r_{n}(t)}{p_{n}}+u_{n}(t)\right] .
$$

Here $p_{n}$ are defined in (11),

$$
r_{n}(t)=\frac{1}{n !}\left(\frac{1}{2}+t\right)\left(\frac{3}{2}+t\right) \ldots\left[\left(n+\frac{1}{2}\right)+t\right],
$$


and

$$
u_{0}(t)=-2 t ; \quad u_{n}(t)=-\frac{t}{n !} \frac{\left(\frac{1}{2}+t\right)\left(\frac{3}{2}+t\right) \ldots\left[\left(n-\frac{1}{2}\right)+t\right]}{\left(n+\frac{1}{2}\right)}, \quad(n=1,2, \ldots) .
$$

The expression (16) applies for

$$
t<+\frac{1}{2}
$$

To put it into Cauchy's form we note that the series

$$
\sum_{n=0}^{\infty} u_{n}(t)
$$

under the condition (17) converges by itself and, hence, can be summed independently. The resulting expression for the amplitude $A(s, t)$ reads

$$
A(s, t)=\frac{\Gamma\left(\frac{1}{2}\right) \Gamma\left(\frac{1}{2}-t\right)}{\Gamma(-t)}+\sum_{n=0}^{\infty}\left[\frac{r_{n}(t)}{s-p_{n}}+\frac{r_{n}(t)}{p_{n}}\right] .
$$

This is precisely the desired Cauchy's form valid for $t<+\frac{1}{2}$. It is easy to check that at $t=0$ the expression (19) consisted with (15). In contrast with (18), the series of correcting polynomials

$$
\sum_{n=0}^{\infty} h_{n}^{(0)}(t)=\sum_{n=0}^{\infty} \frac{r_{n}(t)}{p_{n}}
$$

diverges at $t>-\frac{1}{2}$ and could not be summed separately.

Now we can trace in more detail what happens with (19) when $t$ crosses the boundary value $t=-\frac{1}{2}$ corresponding to the change of the asymptotic regime $A(s, t) \sim s^{\alpha_{1}(t)}$ from $\alpha_{1}(t) \geq 0$ to $\alpha_{1}(t)<0$. It can be easily shown that at $t<-\frac{1}{2}$ the series (20) converges, the summation giving the result (cf. with (8))

$$
\sum_{n=0}^{\infty} h_{n}^{(0)}(t)=-\frac{\Gamma\left(\frac{1}{2}\right) \Gamma\left(\frac{1}{2}-t\right)}{\Gamma(-t)}, \quad\left(t<-\frac{1}{2}\right) .
$$

Thus, in complete accordance with the general scheme discussed above (see Sec. 3), we conclude that at $t<-\frac{1}{2}$ the amplitude (10) admits a representation

$$
A(s, t)=\sum_{n=0}^{\infty} \frac{r_{n}(t)}{s-p_{n}}
$$

constructed solely from the resonance contributions; at $t=-1$ this form coincides with (15). The expression (21) is oftenly used in the literature to show the physical content of the string amplitude (10). 
The important conclusion to be drawn from the above analysis is that the conventional dual (string) models of hadrons are based on three rather different general postulates, to say nothing about suggestions of a particular nature. First, they take advantage of the crossing symmetry requirement. Second, they are rested on certain analyticity conditions, namely, those of meromorphy and polynomial boundedness. Third, they imply - though in a highly latent form - a particular suggestion about the (unique!) connection between the direct channel spectrum parameters and the point-like (background) interactions, this connection explicitly revealing only in the band of the momentum transfer $t$ corresponding to $\alpha_{I}(0) \geq 0$.

The two first postulates are quite general, whereas the third one is nothing but an artifact of the ansatz based on $B$-functions. Thus it looks reasonable to consider a theory which is free of any particular suggestions about the structure of point-like vertices. This is precisely the way which we follow here.

\section{Bootstrap equations for the parameters of $(\pi, K)$ resonances}

Let us now turn to a consideration of $(\pi, K)$ processes. Unlike [1], here we are interested mostly in derivation of the complete set of bootstrap constraints. For this we use the Cauchy forms in the bands $B_{s}, B_{t}$ and $B_{u}$ corresponding to three cross-conjugated channels. In contrast with [1], we write those forms in terms of independent pairs of kinematical variables $\left\{\nu_{x}, x\right\} \quad(x=s, t, u)$ and impose more realistic - Regge - asymptotic requirements. Since the logical scheme, compared to that described in [1], remains unchanged, we omit unnecessary comments. The summary of relevant formulae and notations is given in Appendix (see also [1]); Fig. 2 explains the geography of bands $B_{x}$ and domains $D_{x}$.

Let us begin our analysis from the band $B_{s}$. In this case $\nu_{s}=(t-u)$ is considered as a complex variable, while $s-$ as a small real parameter $(|s| \sim 0)$. So, the Cauchy form (5) for the combination $(A+2 B)_{B_{s}}$ with the principal parts defined according to (73) and (74) (under the condition (70) fixing the bounding polynomial degree $N=0$ ) reads

$$
\begin{aligned}
(A+2 B)_{B_{s}}=\alpha_{s}(s) & +2 \sum_{(I=0)} G_{0} P_{J}\left(\frac{\Sigma+2 s}{4 F}\right)\left\{\frac{1}{\nu_{s}+(s+2 \theta)}-\frac{1}{s+2 \theta}\right\} \\
& +4 \sum_{(I=1)} G_{1} P_{J}\left(\frac{\Sigma+2 s}{4 F}\right)\left\{\frac{1}{\nu_{s}+(s+2 \theta)}-\frac{1}{s+2 \theta}\right\}
\end{aligned}
$$




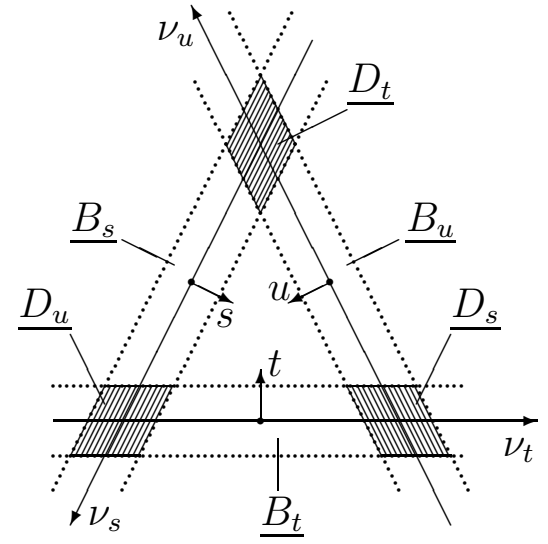

Figure 2: Disposition of the bands $B_{x}$ and intersection domains $D_{x}(x=s, t, u)$.

$$
+2 \sum_{(I=1 / 2)} G_{1 / 2} P_{J}\left(1-\frac{\Sigma+s}{2 \Phi}\right)\left\{\frac{1}{\nu_{s}-(s+2 \theta)}+\frac{1}{s+2 \theta}\right\} .
$$

Here $\alpha_{s}$ stands for unknown function of $s$.

The analogous form for $(A-B)_{B_{s}}$ reads

$$
\begin{aligned}
(A-B)_{B_{s}}= & 2 \sum_{(I=0)} G_{0} P_{J}\left(\frac{\Sigma+2 s}{4 F}\right) \frac{1}{\nu_{s}+(s+2 \theta)} \\
& -2 \sum_{(I=1)} G_{1} P_{J}\left(\frac{\Sigma+2 s}{4 F}\right) \frac{1}{\nu_{s}+(s+2 \theta)} \\
& -4 \sum_{(I=1 / 2)} G_{1 / 2} P_{J}\left(1-\frac{\Sigma+s}{2 \Phi}\right) \frac{1}{\nu_{s}-(s+2 \theta)} .
\end{aligned}
$$

In the latter case no unspecified functions of $t$ appear in the Cauchy form because according to (70) - the degree of the relevant bounding polynomial is negative $(N=-1)$. From (22) and (23) one derives

$$
(A)_{B_{s}}=\frac{1}{3} \alpha_{s}(s)+2 \sum_{(I=0)} G_{0} P_{J}\left(\frac{\Sigma+2 s}{4 F}\right)\left\{\frac{1}{\nu_{s}+(s+2 \theta)}-\frac{1}{3} \frac{1}{s+2 \theta}\right\}
$$




$$
\begin{array}{r}
-\frac{4}{3} \sum_{(I=1)} G_{1} P_{J}\left(\frac{\Sigma+2 s}{4 F}\right) \frac{1}{s+2 \theta} \\
-2 \sum_{(I=1 / 2)} G_{1 / 2} P_{J}\left(1-\frac{\Sigma+s}{2 \Phi}\right)\left\{\frac{1}{\nu_{s}-(s+2 \theta)}-\frac{1}{3} \frac{1}{s+2 \theta}\right\}
\end{array}
$$

and

$$
\begin{aligned}
(B)_{B_{s}} & =\frac{1}{3} \alpha_{s}(s)-\frac{2}{3} \sum_{(I=0)} G_{0} P_{J}\left(\frac{\Sigma+2 s}{4 F}\right) \frac{1}{s+2 \theta} \\
& +2 \sum_{(I=1)} G_{1} P_{J}\left(\frac{\Sigma+2 s}{4 F}\right)\left\{\frac{1}{\nu_{s}+(s+2 \theta)}-\frac{2}{3} \frac{1}{s+2 \theta}\right\} \\
& +2 \sum_{(I=1 / 2)} G_{1 / 2} P_{J}\left(1-\frac{\Sigma+s}{2 \Phi}\right)\left\{\frac{1}{\nu_{s}-(s+2 \theta)}+\frac{1}{3} \frac{1}{s+2 \theta}\right\} .
\end{aligned}
$$

The similar consideration in the band $B_{t}$ results in the expressions

$$
\begin{gathered}
(A)_{B_{t}}=a_{0}(t)-2 \sum_{(I=1 / 2)} G_{1 / 2} P_{J}\left(1+\frac{t}{2 \Phi}\right)\left\{\frac{1}{\nu_{t}-(t+2 \theta)}-\frac{1}{\nu_{t}+(t+2 \theta)}+\frac{2}{t+2 \theta}\right\}, \\
(B)_{B_{t}}=-2 \sum_{(I=1 / 2)} G_{1 / 2} P_{J}\left(1+\frac{t}{2 \Phi}\right)\left\{\frac{1}{\nu_{t}-(t+2 \theta)}+\frac{1}{\nu_{t}+(t+2 \theta)}\right\} .
\end{gathered}
$$

Here $a_{0}(t)$ is another unknown function of $t$.

At last, in $B_{u}$ one has

$$
\begin{aligned}
(A)_{B_{u}}= & \frac{1}{3} \alpha_{u}(u)-2 \sum_{(I=0)} G_{0} P_{J}\left(\frac{\Sigma+2 u}{4 F}\right)\left\{\frac{1}{\nu_{u}+(u+2 \theta)}-\frac{1}{3} \frac{1}{u+2 \theta}\right\} \\
- & \frac{4}{3} \sum_{(I=1)} G_{1} P_{J}\left(\frac{\Sigma+2 u}{4 F}\right) \frac{1}{u+2 \theta} \\
+ & 2 \sum_{(I=1 / 2)} G_{1 / 2} P_{J}\left(1-\frac{\Sigma+u}{2 \Phi}\right)\left\{\frac{1}{\nu_{u}+(u+2 \theta)}+\frac{1}{3} \frac{1}{u+2 \theta}\right\}, \\
(B)_{B_{u}}= & -\frac{1}{3} \alpha_{u}(u)+\frac{2}{3} \sum_{(I=0)} G_{0} P_{J}\left(\frac{\Sigma+2 u}{4 F}\right) \frac{1}{u+2 \theta} \\
& +2 \sum_{(I=1)} G_{1} P_{J}\left(\frac{\Sigma+2 u}{4 F}\right)\left\{\frac{1}{\nu_{u}-(u+2 \theta)}+\frac{2}{3} \frac{1}{u+2 \theta}\right\} \\
& +2 \sum_{(I=1 / 2)} G_{1 / 2} P_{J}\left(1-\frac{\Sigma+u}{2 \Phi}\right)\left\{\frac{1}{\nu_{u}+(u+2 \theta)}-\frac{1}{3} \frac{1}{u+2 \theta}\right\},
\end{aligned}
$$


where $\alpha_{u}(u)$ is the third unknown function.

The system of relations (24) - (29) exhausts constructive potentialities of our postulates. The further progress can be only based on the analysis of this system.

First, we need to specify the form of three unknown functions $a_{0}(t), \alpha_{s}(s)$ and $\alpha_{u}(u)$ in terms of the resonance spectrum parameters. Precisely as in [1], this can be done through a comparison of the pairs of relevant forms in the domains of mutual intersection of the corresponding bands. Thus we have to analyze the following conditions $(X=A, B)$ :

$$
\begin{array}{ll}
X\left(M / B_{t}\right)=X\left(M / B_{u}\right), & M \in D_{s}=B_{t} \cap B_{u} ; \\
X\left(M / B_{u}\right)=X\left(M / B_{s}\right), & M \in D_{t}=B_{s} \cap B_{s} ; \\
X\left(M / B_{s}\right)=X\left(M / B_{B_{t}}\right), & M \in D_{u}=B_{s} \cap B_{t} .
\end{array}
$$

The analysis is simple though tedious. Therefore, we give here only general outline and show the final results.

To present our results in a compact form we introduce three auxiliary functions depending on two real variables $x$ and $\nu$. They are the following

$$
\begin{aligned}
& \Phi(x, \nu) \stackrel{\text { def }}{=}-\sum_{(I=0)} G_{0} \frac{P_{J}\left(\frac{\nu+x-M^{2}}{4 F}\right)}{x-M^{2}}-\sum_{(I=1)} G_{1} \frac{P_{J}\left(\frac{\nu+x-M^{2}}{4 F}\right)}{x-M^{2}} \\
&-4 \sum_{\left(I=\frac{1}{2}\right)} G_{1 / 2}\left[\frac{P_{J}\left(1+\frac{\nu+x-2 \theta}{4 \Phi}\right)-P_{J}\left(1+\frac{x}{2 \Phi}\right)}{\nu-(x+2 \theta)}-\frac{P_{J}\left(1+\frac{x}{2 \Phi}\right)}{x+2 \theta}\right], \\
& \Psi_{1}(x, \nu) \stackrel{\text { def }}{=} 3\left\{\sum_{(I=0)} G_{0}\left[\frac{P_{J}\left(\frac{\Sigma+2 x}{4 F}\right)-P_{J}\left(\frac{\nu+x-M^{2}}{4 F}\right)}{\nu-(x+2 \theta)}+\frac{2}{3} \frac{P_{J}\left(\frac{\Sigma+2 x}{4 F}\right)}{x+2 \theta}\right]\right. \\
&+\sum_{(I=1)} G_{1}\left[\frac{P_{J}\left(\frac{\Sigma+2 x}{4 F}\right)-P_{J}\left(\frac{\nu+x-M^{2}}{4 F}\right)}{\nu-(x+2 \theta)}+\frac{4}{3} \frac{P_{J}\left(\frac{\Sigma+2 x}{4 F}\right)}{x+2 \theta}\right] \\
&\left.-\sum_{\left(I=\frac{1}{2}\right)} G_{1 / 2}\left[\frac{P_{J}\left(1+\frac{\nu+x-2 \theta}{4 \Phi}\right)}{x-M^{2}}+\frac{2}{3} \frac{P_{J}\left(1-\frac{\Sigma+x}{2 \Phi}\right)}{x+2 \theta}\right]\right\} \\
& \Psi_{2}(x, \nu) \stackrel{\text { def }}{=} 2 \sum_{(I=0)} G_{0} \frac{P_{J}\left(\frac{\Sigma+2 x}{4 F}\right)}{x+2 \theta}-6 \sum_{(I=1)} G_{1} P_{J}\left(\frac{\Sigma+2 x}{4 F}\right)\left[\frac{1}{\nu+(x+2 \theta)}-\frac{2}{3} \frac{1}{x+2 \theta}\right]
\end{aligned}
$$




$$
\begin{aligned}
-3 \sum_{I=\frac{1}{2}} G_{1 / 2}[ & 2 \frac{P_{J}\left(1-\frac{\Sigma+x}{2 \Phi}\right)-P_{J}\left(1-\frac{\nu+x-2 \sigma}{4 \Phi}\right)}{\nu-(x+2 \theta)} \\
& \left.+\frac{2}{3} \frac{P_{J}\left(1-\frac{\Sigma+x}{2 \Phi}\right)}{x+2 \theta}+\frac{P_{J}\left(1-\frac{\nu+x-2 \sigma}{4 \Phi}\right)}{x-M^{2}}\right] .
\end{aligned}
$$

Each of the above constructions presents well-defined expression near the point $(x=0$, $\nu=2 \sigma)$. This is not an assumption: the expressions (33)-(35) appear naturally during the analysis of consistency requirements (30)-(32), and their convergency follows directly from our asymptotic conditions.

Let us start the consideration from the domain $D_{s}$. The first of the conditions (30), namely,

$$
B /{ }_{B_{t}}=B / B_{u}
$$

gives

$$
\alpha_{u}(u)=\Psi_{2}\left(u,-\nu_{u}\right), \quad\left(u \sim 0, \nu_{u} \sim-2 \sigma\right)
$$

The second independent condition (30)

$$
(A+B) /_{B_{t}}=(A+B) / B_{u}
$$

results in the equality

$$
a_{0}(t)=\Phi\left(t, \nu_{t}\right), \quad\left(t \sim 0, \nu_{t} \sim 2 \sigma\right) .
$$

No other conclusions can be drawn from the condition (30) and the expressions (26)-(29).

Similarly, the analysis of the condition (31) results in the following expressions for $\alpha_{s}(s)$ and $\alpha_{u}(u)$ in $D_{t}$

$$
\begin{array}{cc}
\alpha_{s}(s)=\Psi_{1}\left(s,-\nu_{s}\right), & \left(s \sim 0, \nu_{s} \sim-2 \sigma\right) . \\
\alpha_{u}(u)=\Psi_{1}\left(u, \nu_{u}\right), & \left(u \sim 0, \nu_{u} \sim 2 \sigma\right) .
\end{array}
$$

At last, from (32) it follows that in $D_{u}$

$$
\begin{array}{ll}
a_{0}(t)=\Phi\left(t,-\nu_{t}\right), & \left(t \sim 0, \nu_{t} \sim-2 \sigma\right) . \\
\alpha_{s}(s)=\Psi_{2}\left(s, \nu_{s}\right), & \left(s \sim 0, \nu_{s} \sim 2 \sigma\right) .
\end{array}
$$


The relations (36)-(41) are the only restrictions following from the compatibility conditions (30)-(32) for the amplitudes $A$ and $B$ defined by the forms (24)-(25) in $B_{s}$, (26) $-(27)$ in $B_{t}$ and (28)-(29) in $B_{u}$.

To proceed further, it is convenient to separate the restrictions (36)-(41) into two independent groups. Noting, that each of the functions $a_{0}(t), \alpha_{s}(s)$ and $\alpha_{u}(u)$ depends only on one variable, we conclude that the dependence of $\Phi\left(x, \nu_{x}\right), \Psi_{1}\left(x, \nu_{x}\right)$ and $\Psi_{2}\left(x, \nu_{x}\right)$ on $\nu_{x}$ is purely fictitious. In other words, to compute the left hand sides of (36) - (41) one can assign to $\nu_{s}, \nu_{t}, \nu_{u}$ any arbitrary values from the validity domain $\nu_{x} \sim 2 \sigma$ of the forms (33)-(35).

The above note allows one to rewrite (36)-(41) in the form of two independent systems. The first one reads

$$
\begin{aligned}
a_{0}(t) & =\Phi(t, 2 \sigma), \\
\alpha_{s}(s) & =\Psi_{1}(s, 2 \sigma), \\
\alpha_{u}(u) & =\Psi_{1}(u, 2 \sigma) .
\end{aligned}
$$

It provides the desired explicit formulae expressing the functions $a_{0}(t), \alpha_{s}(s)$ and $\alpha_{u}(u)$ in terms of the resonance spectrum parameters: triple couplings $G_{I}$ and masses $M^{2}, \mu^{2}, m^{2}$.

The second group consists of infinite set of self-consistency conditions. It reads

$$
\begin{gathered}
\frac{\partial^{k+p} \Psi_{1}(x, \nu)}{\partial x^{k} \partial \nu^{p}} /_{Q}=\frac{\partial^{k+p} \Psi_{2}(x, \nu)}{\partial x^{k} \partial \nu^{p}} /_{Q} ; \\
\frac{\partial^{k+p+1} \Psi_{2}(x, \nu)}{\partial x^{k} \partial \nu^{p+1}} /_{Q}=0 ; \\
\frac{\partial^{k+p+1} \Phi(x, \nu)}{\partial x^{k} \partial \nu^{p+1}} /_{Q}=0 ; \\
Q \equiv\{x=0, \nu=2 \sigma\} ; \quad k, p=0,1,2 \ldots .
\end{gathered}
$$

These conditions follow directly from the noted above independence of $\Phi(x, \nu), \Psi_{1}(x, \nu)$ and $\Psi_{2}(x, \nu)$ of the second argument and from the equivalence of (38) and (41). Later on we call this property as reparameterization invariance (RP-invariance). The expressions (33)-(35) provide the explicit forms of the generating functions for the system (43).

RP-invariance imposes very strong constraints on the values of spectrum parameters. Those constraints mirror the general properties of analyticity and crossing symmetry. In the literature they are commonly called as bootstrap. 


\section{Analysis of the bootstrap constraints}

It would be the best if we could show the closed solution of the system (43). Unfortunately, we cannot. This is not only connected with our inability to solve this infinite system, but also with the obvious necessity to get a deeper understanding of the form of each individual equation. Therefore, below we concentrate mostly on the detailed semiphenomenological analysis of few "lowest" equations corresponding to $k, p \leq 1$ in (43).

However, before starting this analysis we would like to point out one important feature of the system (43). Using the expressions (33)-(35) for the generating functions $\Phi, \Psi_{1}$ and $\Psi_{2}$ one can easily show that the scalar particles (both with $I=0$ and $I=1 / 2$ ) do not contribute to this system at all. Thus no limitations on their couplings and masses follow from (43). This feature is uniquely connected with the local character of our consideration. We do not use any assumptions about the asymptotic behavior of the amplitude outside the narrow band corresponding to the momentum transfer close to zero. Compared to [1], we use here much weaker (Regge) asymptotic restrictions which do not require the decreasing behavior of the amplitudes of nonexotic channels. This very reason explains the difference between the corresponding systems of bootstrap constraints.

The above note clearly shows that - as for now - it would be premature to seek the general solution of the system (43); additional information concerning the asymptotic behavior at larger values of the momentum transfer should be first taken into account.

Let us now turn to a consideration of the lowest order bootstrap constraints (43). Those constraints look too bulky to be shown here explicitly. Therefore, below we show their form in $S U_{3}$ chiral limit $m^{2}=\mu^{2}=0$. The only exception will be made for the relation

$$
\Psi_{1}(0,2 \sigma)=\Psi_{2}(0,2 \sigma)
$$

from the first group of the Eqs. (43). With the help of (33) and (35) it can be written in the form

$$
\sum_{(I=1)} \sum_{J=1,3, \ldots} \frac{G_{1}}{M^{2} \Sigma} P_{J}\left(\frac{\Sigma}{4 F}\right)=\sum_{\left(I=\frac{1}{2}\right)} \sum_{J=1,2, \ldots} \frac{G_{1 / 2}}{M^{2} \Sigma}\left[1-P_{J}\left(1-\frac{\Sigma}{2 \Phi}\right)\right]
$$

which is quite suitable for the numerical testing.

By construction (see Sec. 3) we have to carry out the summation in (45) in order of increasing mass. Thus the contributions of the lightest mesons ( $\rho$ and $K^{*}$ ) can be separated without breaking the convergence of the remaining series (such a trick with 
respect to the lowest spin $J=1$ contributions would be a mistake!). This gives

$$
\frac{G_{\rho}}{M_{\rho}^{2} F_{\rho}}+\ldots=\frac{2 G_{K^{*}}}{M_{K^{*}}^{2} \Phi_{K^{*}}}+\ldots
$$

where ellipsis stand for the contribution due to heavier $(M \geq 1.4 \mathrm{GeV})$ mesons. Using the experimental data [14] and the expressions for $F$ and $\Phi$ (see Appendix) along with the $S U_{3}$ estimate for $G_{\rho}$

$$
\frac{G_{\rho}}{F_{\rho}}=\frac{2 G_{K^{*}}}{\Phi_{K^{*}}}
$$

one obtains (in $\mathrm{GeV}^{-2}$ )

$$
(32 \pm 2)+\ldots=(24 \pm 1.2)+\ldots
$$

The agreement does not look satisfactory. The reason becomes clear when we take account of the contributions due to other relatively light mesons listed in [14] (with $M \leq 2 \mathrm{GeV}$ ). In this case we obtain the relation (see Appendix, Table 1)

$$
(34.5 \pm 2.5)+\ldots=(29.5 \pm 2.5)+\ldots,
$$

which looks much more impressive. It is easy to understand that the contributions of heavy mesons $(M \geq 2 \mathrm{GeV})$ cannot destroy the agreement. Indeed, for those mesons $2 \sigma \ll M^{2}$ and, hence, we can use the limit $\sigma=0$ when computing the corresponding terms. In this case

$$
P_{J}\left(\frac{\Sigma}{4 F}\right)=P_{J}(1)=1, \quad P_{J}\left(1-\frac{\Sigma}{2 \Phi}\right)=P_{J}(-1)=(-1)^{J}
$$

and we can rewrite (45) as follows

$$
\begin{aligned}
& (34.5 \pm 2.5)+\sum_{(I=1)} \sum_{J=1,3, \ldots} \frac{G_{1}}{M^{4}} /_{M^{2}>2 \mathrm{GeV}}= \\
& (29.5 \pm 2.5)+\sum_{\left(I=\frac{1}{2}\right)} \sum_{J=1,3, \ldots} \frac{2 G_{1 / 2}}{M^{4}} /_{M^{2}>2 \mathrm{GeV}}
\end{aligned}
$$

where the sum in the right hand side does not contain contributions of mesons with even spins at all. The (approximate) equivalence of the sums in (48) follows directly from $S_{3}$ symmetry.

The above analysis shows that the relative magnitude of two most significant contributions - those of $\rho$ - and $K^{*}$-mesons - appearing in the lowest order bootstrap condition 
(44) (based on the Regge asymptotic requirements), proves to be quite consistent with the well established experimental data. This conclusion remains also true with respect to the constraints of higher orders. In particular, the corresponding relation of the next - compared to (45) — order follows from the second group of bootstrap constraints (43) at $k=1, p=0$. In the limit $m^{2}=\mu^{2}=0$ it reads

$$
\sum_{I=1} \frac{G_{1}}{M_{1}^{6}}[J(J+1)-1]=\sum_{I=1 / 2} \frac{G_{1 / 2}}{M^{6}}\left[1-(-1)^{J}\right][J(J+1)-1]
$$

the correctness of the relationship among the contributions of mesons with $I=1 / 2$ and $I=1$ being obvious. However the numerical test of the SR (49) is of less interest compared to that of (45) since in this case the contributions of mesons with $J \geq 2$ prove to be relatively more important.

Among the constrains (43) there are also sum rules containing the contributions of isoscalar mesons with $J=2,4, \ldots$. For example, the relation

$$
-\sum_{I=0} \frac{G_{0}}{M^{4}} J(J+1)+\sum_{I=1} \frac{G_{1}}{M^{4}} J(J+1)=\sum_{I=1 / 2} \frac{2 G_{1 / 2}}{M^{4}}\left\{\left[1-(-1)^{J}\right]+(-1)^{J} J(J+1)\right\}(
$$

can be derived either from the first group of (43) at $k+p=1$ or from the third one at $k+p=0$.

Unfortunately, as in the case of Eq. (49), the existing data on $\pi K$ resonances are not sufficient for the reliable numerical analysis of $(50)$.

The system of bootstrap constraints (43) is based on the Regge asymptotic requirements. It differs from the analogous system following from the much stronger asymptotic conditions considered in [价. This difference, in turn, corresponds to different forms of the function $a_{0}(t)$ appearing in the expression (26) for the amplitude of elastic $\pi K$ scattering. To compute chiral coefficients we have to make a choice between two possibilities. The phenomenological analysis provides arguments in favor of the Regge-like bootstrap. Therefore, when calculating LEC's in the next Section we use the expression (37) instead of that given in [1].

\section{Low energy coefficients, chiral duality and light scalars}

Now we have all necessary ingredients to express the low energy coefficients of the elastic scattering amplitudes $A\left(\nu_{t}, t\right)$ and $B\left(\nu_{t}, t\right)$ in terms of the spectrum parameters 
$G_{I}, M^{2}, m^{2}$ and $\mu^{2}$. For this we need the expressions (26) and (27) along with the bootstrap requirements (37) and (43) allowing one to fix the form of $a_{0}(t)$. To simplify the form of $A\left(\nu_{t}, t\right)$ it is appropriate to choose the parameterization $\nu=2 \sigma-t$ in (37), this choice being permissible since we are only interested in the values of $t$ close to zero.

The resulting Cauchy forms for $A\left(\nu_{t}, t\right)$ and $B\left(\nu_{t}, t\right)$

$$
\begin{aligned}
A(\nu, t)=- & \sum_{(I=0)} G_{0} \frac{P_{J}\left(\frac{\Sigma}{4 F}\right)}{t-M_{0}^{2}}+\sum_{(I=1)} G_{1} \frac{P_{J}\left(\frac{\Sigma}{4 F}\right)}{t-M_{1}^{2}} \\
+2 \sum_{I=1 / 2} G_{1 / 2}\left\{P_{J}\left(1+\frac{t}{2 \Phi}\right)\left[\frac{1}{\nu+(t+2 \theta)}-\frac{1}{\nu-(t+2 \theta)}\right]\right. & \left.+\frac{P_{J}\left(1-\frac{\Sigma}{2 \Phi}\right)-P_{J}\left(1+\frac{t}{2 \Phi}\right)}{\Sigma+t}\right\}, \\
B(\nu, t)= & -2 \sum_{I=1 / 2} G_{1 / 2} P_{J}\left(1+\frac{t}{2 \Phi}\right)\left[\frac{1}{\nu+(t+2 \theta)}+\frac{1}{\nu-(t+2 \theta)}\right]
\end{aligned}
$$

converge uniformly near the point $\nu_{t}=0, t=0$. This feature allows one to rewrite them in the form of convergent power series

$$
A(\nu, t)=\sum_{i, j=0}^{\infty} a_{i j} \nu^{i} t^{j}, \quad B(\nu, t)=\sum_{i, j=0}^{\infty} b_{i j} \nu^{i} t^{j}
$$

with the low energy coefficients $a_{i j}$ and $b_{i j}$ completely determined by the parameters $G_{I}, M_{I}, \sigma$ appearing in the right hand sides of (51) and (52). Clearly, owing to the symmetry properties

$$
a_{2 k+1, j}=b_{2 k, j}=0, \quad(j, k=0,1, \ldots) .
$$

Let us first consider the coefficient $b_{10}$. From (52) one obtains

$$
b_{10}=\sum_{(I=1 / 2)} \frac{G_{1 / 2}}{\theta^{2}} \text {. }
$$

Chiral $S U_{2} \times S U_{2}$ symmetry tells us that at $\mu=0$ the left hand side of (55) is equal to $1 /\left(4 f_{\pi}^{2}\right)$. Thus we obtain

$$
\frac{1}{4 f_{\pi}^{2}}=\sum_{(I=1 / 2)} \frac{G_{1 / 2}}{M^{2}-m^{2}}
$$


where both sides should be computed at $\mu^{2}=0$. However, the pion mass is very small and to get an estimate one can take the physical values of the parameters in the rhs of the relation (56). This gives (in units of $\mathrm{GeV}^{-2}$ ):

$$
33.0=(20 \pm 2.0)+\ldots
$$

The difference between two sides in (57) is too large to be explained by the corrections connected with the pion mass. The second line of the Table 1 shows that it also cannot be attributed to slow convergence of SR (56).

Natural solution to this problem is provided by suggestion on the existence of a relatively light resonance (or, perhaps, two ones) with $I=1 / 2$. It must be a scalar, because otherwise the correct balance in SR (48) would be disturbed. This resonance is known as $\kappa$-meson. It appears in various theoretical schemes as well as in results of the analysis of experimental data (K-matrix, Padé-approximants, etc; see [15], 14] and previous review issues by PDG). The current status of $\kappa$-meson is even less clear than that of $\sigma$-meson. It should be noted, however, that - after a long hiatus interest in both particles has quickened in the past few years. Many authors (see 16] - [23]) have reanalized the problem of light scalars from rather different viewpoints (potential models, unitarized resonance models, K-matrix analysis, chiral symmetry, etc) and concluded that light broad scalar mesons do exist, though their parameters (masses and coupling constants) still cannot be fixed with sufficient accuracy. Further theoretical and experimental efforts are needed to clarify the situation in scalar sector.

Since the SR (56) follows from rather general postulates, we can use it to estimate the $\kappa$-meson parameters. Based on the assumption that there is only one light scalar with the mass $M \leq 1.4 \mathrm{GeV}$ one obtains from (56) and (57) the following (rough) estimate

$$
\frac{G_{\kappa}}{\left(M_{\kappa}^{2}-m^{2}\right)^{2}} \sim 10 \mathrm{GeV}^{-2}
$$

Using this estimate and the expression (77) for $G_{1 / 2}^{(0)}$ one concludes that $\kappa$-meson with $M_{\kappa}=1 \mathrm{GeV}$ would have the width $\Gamma_{\pi K} \approx 220 \mathrm{MeV}$ while $M_{\kappa}=1.4 \mathrm{GeV}$ would correspond to $\Gamma_{\pi K} \approx 1 \mathrm{GeV}$. In what follows we assume that

$$
M_{\kappa} \approx 1 \mathrm{GeV}
$$

This value should not be taken too seriously: it provides only indicative numbers. Light scalar mesons - if exist - are broad; in such a case the very meaning of the term "width" loses its definiteness. We imply the meaning suggested by the relations (75) - (77).

The relations (58) - (59) allow one to estimate the magnitudes of the $\kappa$-meson contributions to numerical values of the coefficients $a_{i j}$ and $b_{i j}$. Let us consider first the 
latter ones. From (52) one derives

$$
\begin{aligned}
& b_{11}=-\sum_{(I=1 / 2)} \frac{G_{1 / 2}^{(J)}}{\theta^{3}}\left[1-\pi_{J}^{(1)} \xi\right], \\
& b_{12}=\sum_{(I=1 / 2)} \frac{G_{1 / 2}^{(J)}}{\theta^{4}}\left[\frac{3}{4}-\pi_{J}^{(1)} \xi+\pi_{J}^{(2)} \xi^{2}\right], \\
& b_{30}=\frac{1}{4} \sum_{(I=1 / 2)} \frac{G_{1 / 2}^{(J)}}{\theta^{4}},
\end{aligned}
$$

where

$$
\pi_{J}^{(k)} \equiv \frac{1}{2^{k}(k !)^{2}} \frac{(J+k) !}{(J-k) !}, \quad(k \leq J) ; \quad \pi_{J}^{(k)}=0, \quad(k>J)
$$

and

$$
\xi=\frac{\theta}{2 \Phi}
$$

The corresponding numerical values can be obtained with the help of data [14] and the estimates (58), (59). They are the following

$$
b_{11}=(53.5 \pm 10) \mathrm{GeV}^{-4} ; \quad b_{12}=(-97 \pm 11) \mathrm{GeV}^{-6} ; \quad b_{30}=(13.5 \pm 2) \mathrm{GeV}^{-6} .
$$

Numerical values of the individual contributions to SR (60) are shown in the Table 1. It is clear that in all three cases the most significant contribution follows from the lightest vector resonance - $K^{*}(892)$, the influence of $\kappa$-meson appearing mainly in the values of error bars. Heavy mesons (those with $M>2 \mathrm{GeV}$ ) play negligible role because the sum rules under consideration possess extremely rapid convergence. The above statements certainly remain true with respect to higher order coefficients $b_{i j}$. Moreover, the values of $b_{i j}$ at $j \neq 0$ - in contrast to those of $b_{j 0}$ — only weakly depend on the assumption (59).

Thus we conclude that chiral VMD (vector meson dominance) hypothesis works satisfactory (with accuracy $\sim 25 \%$ ) for all the coefficients except $b_{10}$, in which case the scalar meson contribution represents more than $30 \%$ of the total value. However, it should be remembered that the validity of this statement strongly depends on the suggestion (59); our conclusion would be quite different if we take $M_{\kappa}=800 \mathrm{MeV}$.

Let us consider now the coefficients $a_{i j}$. From the structure of (51) it follows that at $i \neq 0$ the value of $a_{i j}$ is completely determined by the contributions of mesons with $I=1 / 2$. In particular,

$$
a_{20}=\frac{1}{2} \sum_{I=1 / 2} \frac{G_{1 / 2}^{(J)}}{\theta^{3}}, \quad a_{21}=\frac{1}{4} \sum_{I=1 / 2} \frac{G_{1 / 2}^{(J)}}{\theta^{4}}\left[-3+\pi_{J}^{(1)} \frac{\theta}{\Phi}\right] .
$$


The corresponding numerical values

$$
a_{20}=(17.2 \pm 2.5) \mathrm{GeV}^{-4}, \quad a_{21}=(32.0 \pm 6.8) \mathrm{GeV}^{-6},
$$

are caused mainly by the contribution of $K^{*}(892)$ (see Table 1 ), the latter conclusion being strongly connected with the assumption (59).

A consideration of the Table 1 allows one to understand the reason for applicability of VMD hypothesis in the cases considered above. Because of extremely rapid convergence of SR (60) and (63), the most significant contribution is provided by the lightest resonance. Since the assumed value of $M_{\kappa}$ is larger than $M_{K^{*}}$, the influence of the $\kappa$-meson happens to be weaker than that of $K^{*}(892)$.

The matters are much more complicated with respect to the coefficients $a_{0 j}$. Let us compute the lowest one. From (51) we have

$$
a_{00}=\sum_{I=0} \frac{G_{0}}{M^{2}} P_{J}\left(\frac{\Sigma}{4 F}\right)-\sum_{I=1} \frac{G_{1}}{M^{2}} P_{J}\left(\frac{\Sigma}{4 F}\right)+2 \sum_{I=1 / 2} \frac{G_{1 / 2}}{\Sigma}\left[P_{J}\left(1-\frac{\Sigma}{2 \Phi}\right)-\frac{\sigma}{\theta}\right] .
$$

Chiral $S U_{2} \times S U_{2}$ symmetry tells us that at $\mu=0$

$$
a_{00}=0 .
$$

The latter condition allows one to get an idea on the magnitude of total scalar-isoscalar meson contribution. Using [14] we compute the contributions of resonances with $I=1 / 2$ - the numbers are shown in Table 1. The influence of $\kappa$-meson is estimated just as above; it happens to be relatively less important than in SR (56). Next we compute the contributions of isovectors $\rho(770)(\sim 3.0)$ and $\rho_{3}(1690)(\sim 2.6)$ and spin-2 isoscalar $f_{2}(1270)(\sim 7.3)$. Summing all the numbers (with the most pessimistic values of error bars) one obtains from (65) and (66) the following sum rule

$$
\sum_{I=J=0} \frac{G_{0}^{(0)}}{M^{2}} \approx 53.5 \pm 7.5
$$

This relation clearly demonstrates that chiral VMD does not apply to the coefficient $a_{00}$ : in this case the contribution due to scalar mesons happens to be larger than that of vector ones (cf the numbers in Table 1 with the rhs of (67)). An idea of the required structure of scalar sector can be gained from the estimate of $f_{0}(1300)$ contribution. Taking (see [14]) $M_{f}=1.25 \mathrm{GeV}, \Gamma_{\pi \pi}=0.37 \mathrm{GeV}$ and $\Gamma_{K \bar{K}}=0.03 \mathrm{GeV}$, one obtains the number

$$
\frac{G_{0}^{(0)}\left(f_{0}\right)}{M_{f_{0}}^{2}} \approx 3.2,
$$


which is negligibly small compared to that required by (67). This estimate shows that SR (67) requires the existence of light scalar-isoscalar resonance strongly coupled to both $\pi \pi$ - and $K \bar{K}$-channels. In principle, the mentioned above $\sigma$-meson would be a good candidate for this role. If we take this hypothesis (along with the parameters, taken from the quoted above papers [16] - [23]), the computation of the coefficients $a_{0 j}$ could be easily done. However, it should be kept in mind that the same resonance appears also in processes of $\pi \pi$ and $K \bar{K}$ scattering. Therefore, from the purely theoretical point of view, it is much more interesting to carry out simultaneous analysis of joint system of sum rules in order to get self-consistent results. This analysis is in progress now.

\section{Concluding remarks}

The method of Cauchy's forms described in Sec. 3 allowed us to avoid model dependence of results. Our conclusion concerning the dual properties of tree-level amplitude in effective field theory with infinite number of field species follows directly from certain analyticity requirements (meromorphy and polynomial boundedness) and the requirement of crossing-symmetry. The same is true with respect to the system of bootstrap constraints which appears as necessary and sufficient condition providing feasibility of analytic continuation between the direct- and cross-channel domains. This conclusion eliminates apparent contradiction between the conventional quantum field theory (QFT) approach and that based on the idea of duality (in Ref. 24] this problem is considered from different point of view). Dual amplitude is constructed from the infinite series of direct channel poles, the cross channel ones appearing just as a result of summation of this series and its subsequent analytic continuation to the cross-channel domain. In contrast, the Born approximation in QFT contains both types of poles simultaneously (plus smooth - background - terms corresponding to point-like interactions). This very feature is commonly considered as drastic difference between two approaches.

Our results show that there is logical gap in the above reasoning. Indeed, the dual amplitude contains infinite number of poles corresponding to the states with arbitrarily high values of spin and mass. Therefore, it is natural to compare it with QFT which also contains the infinite spectrum of bound states. Next, as it follows from our analysis in Sec. 4, every dual amplitude along with infinite set of poles contains also specific background terms which manifest themselves explicitly in the corresponding area of the momentum transfer. Thus the QFT in question can also contain pointlike interaction terms. At last, the QFT amplitude - as well as the dual one - should be written in the correct analytic form, the latter one depending on the domain under consideration. In particular, in the direct-channel domain this form cannot contain any poles in momentum 
transfer, the contribution of $t$-channel exchange graphs looking here like the background interaction with infinite number of derivatives. With this understanding in mind, one can write down the most general QFT expression for tree-level amplitude (which is nothing but the amplitude of the effective QFT) in the form dictated by the analyticity requirements, and then carry out the analytic continuation to the cross-channel domain. The conditions guaranteeing that the resulting expression will contain no other singularities but simple poles and ambiguity points, and possess the desired asymptotic behavior, are precisely those expressed by the system of bootstrap constraints.

Thus we conclude that the effective field theory of strong interactions, based on the idea of quark-hadron duality, necessarily results in the string form of tree-level amplitude provided that certain analyticity requirements (meromorphy and polynomial boundedness) are imposed. This conclusion provides a solution to the problem of string organization of field theories [24].

Further, though the mere appearance of bootstrap constraints does not depend of the values of bounding polynomial degrees, their particular form does depend of those values. It is remarkable that numerical test based solely on low energy data provides clear arguments in favor of the degrees corresponding to experimentally known values of intercepts. This means that the formulae for low energy coefficients (Sec. 7), obtained as by-product of our study of effective hadron theory with maximal analyticity, may be thought of as model independent results based on well established general principles. However, it should be kept in mind that, to use those formulae in ChPT computations, one needs to expand them in powers of quark masses. This is necessary just to avoid contradiction with chiral power counting rules.

Our main conclusion concerning the structure of LEC's is the following. The idea of Chiral duality [3, 4, 25, 26] (for the review see [27, 28]) is certainly true. It mirrors the requirements of general principles of quark-hadron duality and analyticity. Thus it is no less general than ChPT itself. However, this idea needs more accurate formulation. Indeed, a comparison of the well-defined form (51) with the formally written expression (73) shows considerable difference in their structure. In contrast with (73), the expression (51) does not contain any unknown polynomials like $E_{A}(s, t, u)$. Instead, it contains the contribution (see the second item) depending on the parameters of isovector resonances; such a term could not appear in the "naive" form $(\sqrt{73})$ in principle. On the other hand, the well-defined form (52) does not contain any contribution from isovectors, while the formal expression (74) does contain it (along with unknown polynomial $E_{B}(s, t, u)$ which is absent in (52)). This means that one should exercise caution when formulating the idea of Chiral duality. In this respect, the situation resembles that with formulation of VMD hypothesis [25, 26, 29] - the latter happened to be well defined only under the condition if certain limitations are imposed on high energy asymptotics of the vector 
meson contribution. In fact, our polynomial boundedness requirement is nothing but a generalized version of those limitations applied to the full tree-level amplitude.

In contrast to Chiral duality, the status of Chiral VMD hypothesis is much less reliable. From recent data analysis and from our SR (65) it follows that the existence of light broad scalar resonance looks necessary to explain the low energy experimental data. In some cases (like $a_{0 j}$ - see Sec. 7) the contribution of this scalar meson may happen to be significant even compared to (also allowed) that of lightest vector mesons. Modern understanding of the scalar sector still looks unsatisfactory.

Here it is a point to stress the difference between our approach and that used by those authors who study various QCD-inspired models (for the review, see [30, 31]) to compute chiral LEC's. Our results show that LEC's can be treated as "secondary" quantities completely fixed by the values of "primary" ones: hadron masses and on-shell coupling constants. The latter values should be taken from the underlying fundamental theory (QCD, string, ...). Given them one can compute all other characteristics of low-energy hadron reactions in a framework of the approach based on effective field theory accounting for few general principles (symmetry and analyticity). In contrast, the authors of QCDinspired models consider LEC's on the same ground as spectrum parameters. Particular assumptions (inavoidable in this approach) concerning the hadronization regime in QCD introduce strong model dependence in the results. This feature along with scarcity of modern database (specially stressed with respect to $(\pi, K)$ processes in [32]) hampers the understanding of relative importance of different mechanisms. That is why we prefer to use the conventional approach [2, 3, 4, supplemented with requirements imposed by the general principle of maximal analyticity.

It should be noted that the latter principle plays no role (or, better, it is trivial) in conventional renormalizable field theories: there is no necessity to postulate anything which can be computed. The necessity of considering maximal analyticity as independent condition appears only if the number of field species (or, the number of derivatives) is allowed to become infinite. As shown recently in [33], in this very case one can expect considerable simplification of a theory near the phase transition point. Therefore, it would be interesting to find an algebraic structure corresponding to the considered above infinite system of bootstrap constraints. It is more or less clear that it might be one of the algebras of rational functions. This suggestion correlates (though indirectly) with the structure of our sum rules which admit existence of infinite-dimensional multiplets.

\section{Acknowledgments}

It is a pleasure to thank A.Andrianov, H.V. von Geramb and M.D.Scadron for the 
information on recent publications and J.Gasser for his interest to this work and friendly support. We are indebted to M.Jarmolovich for her help in preparation of the manuscript.

This work was supported in part by RFBR (Grant 98-02-18137) and by GRACENAS (Grant 6-19-97, 1997). The work of A.Vereshagin was supported also by ISSEP "Soros Students" (Grant s97-2391).

\section{Appendix}

Here we give a summary of formulae and relations which are necessary for the analysis of $(\pi, K)$ processes.

Three different channels of the reaction under consideration are the following

$$
\begin{aligned}
& \pi_{a}\left(k_{1}\right)+K_{\alpha}\left(p_{1}\right) \longrightarrow \pi_{b}\left(k_{2}\right)+K_{\beta}\left(p_{2}\right), \\
& \pi_{a}\left(k_{1}\right)+\frac{\pi_{b}\left(k_{2}\right)}{K_{\alpha}}\left(p_{1}\right)+K_{\beta}\left(p_{2}\right), \\
& \pi_{a}\left(k_{1}\right)+\overline{K_{\beta}}\left(p_{2}\right) \longrightarrow \pi_{b}\left(k_{2}\right)+\overline{K_{\alpha}}\left(p_{1}\right) .
\end{aligned}
$$

Here $a, b=1,2,3$ and $\alpha, \beta=1,2$ stand for isotopic indices. The amplitude can be written as follows

$$
M_{\beta \alpha}^{b a}=\delta_{a}^{b} \delta_{\alpha}^{\beta} A(s, t, u)+i \varepsilon_{b a c}\left(\sigma_{c}\right)_{\beta \alpha} B(s, t, u),
$$

where $\operatorname{Tr}\left(\sigma_{a} \sigma_{b}\right)=2 \delta_{a b}$, and

$$
\begin{gathered}
s=\left(k_{1}+p_{1}\right)^{2}, \quad t=\left(k_{1}-k_{2}\right)^{2}, \quad u=\left(k_{1}-p_{2}\right)^{2}, \\
s+t+u=2\left(m^{2}+\mu^{2}\right) \equiv 2 \sigma .
\end{gathered}
$$

Here $\mu(m)$ is the pion (kaon) mass. Due to requirements of Bose symmetry

$$
A(s, t, u)=A(u, t, s) ; \quad B(s, t, u)=-B(u, t, s)
$$

We use also 3 different pairs of independent kinematical variables $\left\{\nu_{x}, x\right\} \quad(x=s, t, u)$ :

$$
\nu_{s}=u-t ; \quad \nu_{t}=s-u ; \quad \nu_{u}=t-s .
$$

Each set $\left\{\nu_{x}, x\right\}$ forms a natural coordinate system in the 3-dimensional band $B_{x}$ corresponding to small real $x$ and arbitrary complex $\nu_{x}$, the section of $B_{x}$ by Mandelstam plane $\left(\right.$ real s,t,u) resulting in a 2 -dimensional strip $S_{x}$ parallel to the side $\mathrm{x}=0$ of the Mandelstam triangle (see Fig.1). 
The Regge theory prescriptions for the asymptotic behavior of the amplitudes $A$ and $B$ in the bands $B_{s}, B_{t}, B_{u}$ can be summarized as follows:

$$
\begin{aligned}
& B_{s}\left\{\left|\nu_{s}\right| \rightarrow \infty ; s \sim 0\right\}:\left\{\begin{array}{lll}
(A+2 B) / B_{s} \sim \nu_{s}^{\alpha_{1 / 2}(s)} ; & {[N=0] ;} \\
(A-B) /_{B_{s}} \sim \nu_{s}^{\alpha_{3 / 2}(s)} ; & {[N=-1] ;}
\end{array}\right. \\
& B_{t}\left\{\left|\nu_{t}\right| \rightarrow \infty ; t \sim 0\right\}:\left\{\begin{array}{rlrl}
A / B_{t} & \sim \nu_{t}^{\alpha_{0}(t)} ; & & {[N=0] ;} \\
B / B_{t} & \sim \nu_{t}^{\alpha_{1}(t)} ; & {[N=-1] ;}
\end{array}\right. \\
& B_{u}\left\{\left|\nu_{u}\right| \rightarrow \infty ; u \sim 0\right\}: \begin{cases}(A-2 B) / B_{u} \sim \nu_{u}^{\alpha_{1 / 2}(u)} ; & {[N=0] ;} \\
(A+B) / B_{u} \sim \nu_{u}^{\alpha_{3 / 2}(u)} ; & {[N=-1] .}\end{cases}
\end{aligned}
$$

Here we also show in braces the degrees of bounding polynomials needed to construct the corresponding Cauchy forms. Those degrees are uniquely determined by the known intercepts of the leading Regge trajectories with the isospin $I$ :

$$
\alpha_{0}(0)=1 ; \quad \alpha_{1}(0) \approx 0,5 ; \quad \alpha_{1 / 2}(0) \approx 0,3 ; \quad \alpha_{3 / 2}(0)<0 .
$$

For the sake of the reader's convenience, below we give also the formal (i.e. constructed in accordance with "naive" Feynman rules) tree-level expressions for the effective amplitudes $A$ and $B$ appearing in (68).

$$
\begin{aligned}
A(s, t, u)= & -\sum_{I=0} G_{0} \frac{P_{J}\left(\frac{s-u}{4 F}\right)}{t-M^{2}}-\sum_{I=1 / 2} G_{1 / 2} P_{J}\left(1+\frac{t}{2 \Phi}\right)\left\{\frac{1}{s-m^{2}}+\frac{1}{u-m^{2}}\right\} \\
& +E_{a}(s, t, u) . \\
B(s, t, u)= & -\sum_{I=0} G_{1} \frac{P_{J}\left(\frac{s-u}{4 F}\right)}{t-M^{2}}-\sum_{I=1 / 2} G_{1 / 2} P_{J}\left(1+\frac{t}{2 \Phi}\right)\left\{\frac{1}{s-m^{2}}-\frac{1}{u-m^{2}}\right\} \\
& +E_{B}(s, t, u) .
\end{aligned}
$$

Here $E_{A}$ and $E_{B}$ stand for the formal power series in $s, t, u$ obeying the Bose symmetry conditions (69), and

$$
\begin{gathered}
F \equiv F\left(M^{2}, m^{2}, \mu^{2}\right)=\frac{1}{4}\left|\sqrt{\left(M^{2}-4 m^{2}\right)\left(M^{2}-4 \mu^{2}\right)}\right| \\
\Phi \equiv \Phi\left(M^{2}, m^{2}, \mu^{2}\right)=\frac{1}{4 M^{2}}\left|\sqrt{M^{4}+m^{4}+\mu^{4}-2 M^{2} m^{2}-2 M^{2} \mu^{2}-2 m^{2} \mu^{2}}\right| .
\end{gathered}
$$

The explicit formulae expressing the constants $G_{I}$ in terms of the corresponding decay widths look as follows

$$
\left|G_{0}^{(J)}\right|=8 \pi M_{S}^{2}(2 J+1) \sqrt{\frac{1}{3} \frac{\Gamma(S \rightarrow \pi \pi)}{\left|\vec{p}_{\pi}\right|} \frac{\Gamma(S \rightarrow K \bar{K})}{\left|\vec{p}_{\mathrm{K}}\right|}}, \quad\left(M_{S} \geq 2 m\right)
$$




\begin{tabular}{||c||c|c|c|c|c|c|c|l||}
\hline \hline $\begin{array}{c}\text { meson } \\
\text { mass }\end{array}$ & $\begin{array}{c}\kappa \\
1.0\end{array}$ & $\begin{array}{c}K_{0}^{*} \\
1.43\end{array}$ & $\begin{array}{c}K^{*} \\
0.89\end{array}$ & $\begin{array}{l}K^{*} \\
1.41\end{array}$ & $\begin{array}{c}K^{*} \\
1.68\end{array}$ & $\begin{array}{l}K_{2}^{*} \\
1.43\end{array}$ & $K_{3}^{*}$ & $K_{4}^{*}$ \\
\hline \hline $\operatorname{rhs}(45)$ & - & - & 24.0 & 0.54 & 1.31 & 2.60 & 0.60 & 0.28 \\
\hline$b_{10}$ & 10 & 2.36 & 12.1 & 0.42 & 1.75 & 2.18 & 0.87 & 0.43 \\
\hline$b_{11}$ & -13 & -1.30 & 48.9 & 0.31 & 0.76 & 7.17 & 3.67 & 2.23 \\
\hline$b_{12}$ & 12 & 0.55 & -103 & -0.22 & -0.36 & 1.25 & 2.50 & 2.30 \\
\hline$b_{30}$ & 3.6 & 0.18 & 10.8 & 0.03 & 0.07 & 0.17 & 0.03 & 0.01 \\
\hline \hline$a_{20}$ & 5.4 & 0.65 & 11.42 & 0.12 & 0.34 & 0.60 & 0.15 & 0.05 \\
\hline$a_{21}$ & -11 & -0.55 & 35.2 & 0.06 & 0.08 & 1.83 & 1.29 & 0.57 \\
\hline$a_{00}$ & -4.12 & -1.47 & -27.7 & -1.87 & -9.74 & 6.91 & -6.40 & 3.00 \\
\hline
\end{tabular}

Table 1: Separate contributions of $I=\frac{1}{2}$ mesons to SR (45) and LEC's.

$$
\begin{gathered}
\left|G_{1}^{(J)}\right|=8 \pi M_{V}^{2}(2 J+1) \sqrt{\frac{1}{2} \frac{\Gamma(V \rightarrow \pi \pi)}{\left|\vec{p}_{\pi}\right|} \frac{\Gamma(V \rightarrow K \bar{K})}{\left|\vec{p}_{\mathrm{K}}\right|}}, \quad\left(M_{S} \geq 2 m\right) ; \\
G_{1 / 2}^{(J)}=8 \pi M_{R}^{2}(2 J+1) \frac{1}{3} \frac{\Gamma(R \rightarrow \pi K)}{|\vec{p}|}, \quad\left(M_{R} \geq m+\mu\right) .
\end{gathered}
$$

As in the Ref. [1] we use the notations

$$
D_{s}=B_{t} \cap B_{u} ; \quad D_{t}=B_{u} \cap B_{s} ; \quad D_{u}=B_{s} \cap B_{t}
$$

for the mutual domains of various pairs of the bands $B_{x}$ and

$$
\theta \equiv M^{2}-\sigma ; \quad \Sigma \equiv M^{2}-2 \sigma
$$

for two special combinations of masses (here $M$ stands for the resonance mass).

\section{References}

[1] V. Vereshagin, Phys.Rev. D 55, 5349 (1997).

[2] S. Weinberg, Physica A 96, 327 (1979).

[3] J. Gasser, H. Leutwyler, Ann. Phys. (N.Y.) 158, 142 (1984).

[4] J. Gasser, H. Leutwyler, Nucl. Phys. B250, 465 (1985). 
[5] S. Weinberg, The quantum theory of fields (Cambrige University Press, Cambridge, 1995), vv. 1 and 2.

[6] S. Weinberg, Phys. Rev. 177, 2604 (1969).

[7] V. Vereshagin, Nucl. Phys. B55, 621 (1973).

[8] A. Bramon, Riazuddin, M. D. Scadron, J. Phys. G 24, 1 (1998).

[9] A. Bolokhov, A. Manashov, M. Polyakov, V. Vereshagin, Phys. Rev. D 48, 3090 (1993).

[10] B. V. Shabat, Introduction to complex analysis (Nauka, Moscow, 1969). (in Russian).

[11] G. B. Arfken, H. J. Weber. Mathematical methods for physicists (Academic, New York, 1995). 4-th edition.

[12] G. Veneziano, Nuovo Cimento 57A, 190 (1968).

[13] P. H. Frampton, Dual resonance models and superstrings (World Scientific, Singapore, 1986). 2-nd edition.

[14] Particle Data Group, R. M. Barnett et al, Phys. Rev. D 54, 1 (1996).

[15] B. R. Martin, D. Morgan, G. Show, Pion-pion interactions in particle physics (Academic, London, 1976).

[16] V. V. Anisovich et al, Phys. Lett. B 355, 363 (1995).

[17] N. A. Törnqvist, M. Roos, Phys. Rev. Lett. 76, 1575 (1996); ibid, 77, 2333 (1996); ibid, 78, 1604 (1997).

[18] S. Ishida et al, Progr. of Theor. Phys. 95, 745 (1996); ibid, 98, 621 (1997).

[19] M. Svec, Phys. Rev. D 53, 2343 (1996); ibid, 55, 4355 (1997).

[20] M. D. Scadron, hep-ph/9710317 (1997); (to be published in Z. Phys. C, 1998).

[21] M. Sadler, H. V. von Geramb, Phys. Rev. C, 56, 1218 (1997). 
[22] J. A. Oller, E. Oset, J. R. Peláez, Preprint SLAC-PUB-7787; hep-ph/9804209 (1998).

[23] D. Black, A. Faribarz, F. Sannino, J. Schechter, Preprint YCTP-P9-98; SU-4240-678 (1998); hep-ph/9804273 (1998).

[24] Y. J. Feng, C. S. Lam. Phys. Rev. D 50, 7430 (1994).

[25] J. F. Donoghue, C. Ramirez, G. Valencia, Phys. Rev. D 39, 1947 (1989).

[26] G. Ecker, J. Gasser, A. Pich, E. de Rafael, Nucl. Phys. B321, 311 (1989).

[27] G. Ecker, Progr. Part. Nucl. Phys. 58, 563 (1995).

[28] A. Pich, Reports on Progr. in Physics 58, 563 (1995).

[29] G. Ecker, J. Gasser, H. Leutwyler, A. Pich, E. de Rafael, Phys. Lett. B 223, 425 (1989).

[30] J. Bijnens, Phys. Reports 265, 369 (1996).

[31] A. Andrianov, V. Andrianov, D. Ebert, Proc. of 11-th International Workshop QFTHEP'96.

Ed. B. Levtchenko. (MSU Publishing House, Moscow, 1997).

[32] V. Bernard, N. Kaiser, Ulf-G. Meissner, Phys. Rev. D 43, R2757 (1991); Nucl. Phys. B364, 283 (1991).

[33] S. Weinberg, Phys. Rev. D 56, 2303 (1997). 